\title{
Study on the relationship of iodine nutritional status with thyroid function and autoantibodies in pregnant women in Baotou
}

\author{
Yuanyuan Fan ${ }^{1}$, Yalu Wang ${ }^{1}$, Chenhui Li ${ }^{2}$, Ziling Li*1 \\ ${ }^{1}$ Department of Endocrinology, Baogang Hospital, Baotou, Inner Mongolia, China \\ ${ }^{2}$ The Second Military Medical University, Shanghai, China
}

Received: May 28, 2020

DOI: $10.5430 /$ dcc.v7n1p3
Accepted: July 19, 2020

Online Published: July 29, 2020

URL: https://doi.org/10.5430/dcc.v7n1p3

\begin{abstract}
Objective: To investigate the relationship of iodine nutritional status with thyroid function in pregnant women in Baotou, and provide a scientific basis for the guide of reasonable iodine supplementation.

Methods: By use of As-Ce catalysis spectrophotometry, the urinary iodine concentration was determined in 90 women during different periods of pregnancy admitted to Baogang Hospital of Inner Mongolia from January 2017 to December 2017. The determination of thyroid function and the autoantibody level was carried out by electro-chemiluminescence immunoassay (ECLIA).

Results: In the early trimester of pregnancy, pregnant women with iodine deficiency, iodine sufficiency and iodine excess accounted for $36.67 \%, 40.00 \%$ and $23.33 \%$ respectively; in the middle trimester of pregnancy, they accounted for $66.67 \%, 26.67 \%$ and $6.66 \%$ respectively; they made up $40.00 \%, 40.00 \%$ and $20.00 \%$ respectively in the late trimester of pregnancy. The rate of iodine deficiency in women in the middle trimester of pregnancy was significantly higher than that in women in the early and the late trimesters of pregnancy respectively, and the difference was statistically significant $(p<.05)$. The rate of iodine deficiency in pregnant women was positively correlated to the positivity of thyroid peroxidase antibody. Moreover, it had no relationship with the positivity of thyroglobulin antibody. No matter in the pregnant women with iodine deficiency or with iodine excess, the abnormality rate of thyroid function and autoantibodies was significantly higher than that in the pregnant women with iodine sufficiency. The difference was statistically significant $(p<.05)$.

Conclusions: The iodine deficiency in the women in the middle trimester of pregnancy is more severe than that in the women in the early and the late trimesters of pregnancy. Iodine deficiency during pregnancy is positively correlated with the positivity of thyroid peroxidase antibody. Pregnant women with iodine deficiency and iodine excess show a higher abnormality rate of thyroid function and autoantibodies. It is recommended to advocate health education on iodine nutrition to pregnant women, and conduct the dynamic monitoring of urinary iodine concentration and the screening of thyroid function and autoantibodies during pregnancy.
\end{abstract}

Key Words: Iodine nutritional status, Pregnancy, Urinary iodine, Thyroid function

${ }^{*}$ Correspondence: Ziling Li; Email: 3301922766@qq.com; Address: Department of Endocrinology, Baogang Hospital, Baotou, Inner Mongolia 014010, China. 


\section{INTRODUCTION}

Recently, the iodine nutritional status and thyroid function in pregnant women have attracted extensive attention. If iodine deficiency or iodine excess occurs in pregnant women, it will impair their thyroid function and then affect their physical fitness and fetuses. This research is designed to evaluate the iodine nutritional status and thyroid function and detect the level of antibodies in women during different periods of pregnancy, explore the relationship of the iodine nutritional status with thyroid diseases among these pregnant women, provide a theoretical basis for scientific and reasonable iodine supplementation and urinary iodine determination (included in routine pregnancy tests) and prompt a theoretical support and guarantee for the prevention of adverse pregnancy outcomes and the implementation of prenatal and postnatal care.

\section{DATA AND METHODS}

\subsection{Research objects}

Ninety cases of pregnant women who admitted to Baogang Hospital of Inner Mongolia from January 2017 to December of 2017 were chosen and divided into 3 groups according to different periods of pregnancy $(n=30)$. In this research, the course no more than 12 weeks was considered as the early trimester of pregnancy, the course of 13 to 27 weeks was considered as the middle trimester of pregnancy and the course no less than 28 weeks was considered as the late trimester of pregnancy. All subjects were local residents. 3 groups of pregnant women (early pregnancy group, middle pregnancy group and late pregnancy group) shared the same baseline age, and it was comparable. Exclusion standards: 1) patients with a past medical history of thyroid diseases and other autoimmune diseases; 2) patients with a medical history of iodine-containing drugs, iodine-containing nutritions and other drugs affecting thyroid function; 3) patients with a past history of other severe clinical diseases; 4) patients with gestational hypertension, preeclampsia, eclampsia, gestational diabetes mellitus and other severe diseases.

\subsection{Methods}

All candidates were informed of the specific contents, methods and notes about this research in detail. All subjects were required to sign informed contents and complete the corresponding questionnaires. The contents of the questionnaire were made up of general information, health condition, dietary intake for iodine etc.. $6 \mathrm{ml}$ of midstream urine was collected from each subject in order to determine the urinary iodine concentration by use of As-Ce catalysis spectrophotometry. $3 \mathrm{ml}$ of venous blood (fasting blood) was taken from each subject to isolate the serum from the blood sample within $2 \mathrm{~h}$ (rotate speed: 4,000 r/min, centrifuged for $5 \mathrm{~min}$ ). Electro-chemiluminescence immunoassay (ECLIA) (Roche
Automatic Analyzer, German) was applied to the determination of thyroid stimulating hormone (TSH), free thyroxine (FT4), free triiodothyronine (FT3), thyroglobulin antibody $(\mathrm{TgAb})$ and thyroid peroxidase antibody (TPOAb).

\subsection{Evaluation standards and grouping}

1) Iodine nutritional status: according to gestational iodine nutritional standards established by WHO in $2007,{ }^{[1]}$ the urinary iodine concentration $<150 \mu \mathrm{g} / \mathrm{L}$ was considered as iodine deficiency, the concentration ranging from $150 \mu \mathrm{g} / \mathrm{L}$ to $250 \mu \mathrm{g} / \mathrm{L}$ was for iodine sufficiency, the concentration ranging from $250 \mu \mathrm{g} / \mathrm{L}$ to $500 \mu \mathrm{g} / \mathrm{L}$ meant more than adequate iodine, and the concentration no less than $500 \mu \mathrm{g} / \mathrm{L}$ stood for iodine excess. With a view to the above standards, these pregnant women were divided into three groups according to the urinary iodine concentration: iodine deficiency group $(<150 \mu \mathrm{g} / \mathrm{L})$, iodine sufficiency group $(150-250 \mu \mathrm{g} / \mathrm{L})$ and iodine excess group ( $\geq 250 \mu \mathrm{g} / \mathrm{L})$. 2) Thyroid function and thyroid antibodies: they were evaluated according to the standards recommended in "Guideline on diagnosis and management of thyroid diseases during pregnancy and postpartum" established in 2012 $2^{[2]}$ (the detection kits used in our hospital were the same with the kits recommended in this guideline). Normal reference values: TPOAb 0-34 ng/ml, TgAb 0-115 ng/ml; TSH, FT3 and FT4 in the early trimester of pregnancy were 0.05-4.50 mU/L, 3.6-5.6 pmol/L and 11.8$21.0 \mathrm{pmol} / \mathrm{L}$; in the middle trimester of pregnancy, they were 0.03-4.5 mU/L, 3.6-5.2 pmol/L and 10.6-17.6 pmol/L; in the late trimester, they were $0.5-4.5 \mathrm{mU} / \mathrm{L}, 3.5-5.2 \mathrm{pmol} / \mathrm{L}$ and 9.2-16.7 pmol/L respectively. Exceeding the normal values of thyroid function was considered as thyroid dysfunction.

\subsection{Statistical treatment}

SPSS 21.0 software was applied to statistical analysis. The measurement data fitting to normal distribution were represented by mean \pm standard deviation, and $t$-test was applied to the comparison among groups. The categorical data were represented by ratio or percentage, and $\chi^{2}$ was applied to the comparison among groups. Spearman correlation analysis was used in the correlation analysis. The difference $p<.05$ was of statistical significance.

\section{RESUlts}

\subsection{Iodine nutritional status}

According to the determination of the urinary iodine concentration, there was no significant difference in the proportion of iodine excess and iodine sufficiency among pregnant women during different periods of pregnancy $(p>.05)$; iodine deficiency in the pregnant women in the middle trimester of pregnancy accounted for a higher proportion in comparison to the pregnant women in the early and the late trimesters 
of pregnancy $(p<.05)$; there was no significant difference in the proportion of iodine deficiency between the early and the late trimesters. See Table 1 for details.

\subsection{The abnormality rate of thyroid function and au- toantibodies in pregnant women with different io- dine nutritional statuses}

Among 90 cases of pregnant women, there were 43 cases of iodine deficiency, 32 cases of iodine sufficiency and 15 cases of iodine excess. No matter in the pregnant women with iodine deficiency or iodine excess, the abnormality rate of thyroid function and autoantibodies was higher than that in the pregnant women with iodine sufficiency, and the difference was of statistical significance $(p<.05)$. See Table 2 for details.

\subsection{The relationship of the urinary iodine concentration with thyroid antibody positivity and thyroid func- tion}

Spearman correlation analysis was used to analyze the correlation of the urinary iodine concentration to thyroid antibodies (TPOAb and TgAb) and thyroid function (FT3, FT4 and TSH). The results showed that the rate of iodine deficiency in pregnant women was positively correlated to the positivity of the thyroid antibody TPOAb $(r=.547, p=.000)$, and the iodine nutritional status in pregnant women was not correlated to both $\operatorname{TgAb}(r=.205, p=.053)$ and thyroid function $(r=.038, p=.722)$.

Table 1. The comparison in the iodine nutritional status in the early, the middle and the late trimesters of pregnancy, $\mathrm{n}(\%)$

\begin{tabular}{lllll}
\hline Group & $\mathbf{n}$ & Iodine Deficiency & Iodine Sufficiency & Iodine Excess \\
\hline Early Pregnancy Group & 30 & $11(36.67)^{*}$ & $12(40.00)$ & $7(23.33)$ \\
Middle Pregnancy Group & 30 & $20(66.67)$ & $8(26.67)$ & $2(6.66)$ \\
Late Pregnancy Group & 30 & $12(40.00)^{*}$ & $12(40.00)$ & $6(20.00)$ \\
$\chi^{2}$ & & 6.502 & 1.552 & 3.360 \\
$p$ & & .039 & .460 & .186 \\
\hline Note. ${ }^{*}$ In comparison to Middle Pregnancy Group, $p<.05$ & & &
\end{tabular}

Table 2. The correlation of thyroid function and autoantibodies abnormality to the iodine nutritional status, $\mathrm{n}(\%)$

\begin{tabular}{|c|c|c|c|c|c|}
\hline \multirow{2}{*}{ Type } & \multirow{2}{*}{$\mathbf{n}$} & \multicolumn{2}{|c|}{ Thyroid Function } & \multicolumn{2}{|c|}{ Autoantibodies } \\
\hline & & Abnormal & Normal & Abnormal & Normal \\
\hline Iodine Deficiency & 43 & $28(65.12)^{*}$ & $15(34.88)$ & $20(46.51)^{*}$ & $23(53.49)$ \\
\hline Iodine Sufficiency & 32 & $15(46.88)$ & $17(53.12)$ & $5(15.63)$ & $27(84.37)$ \\
\hline Iodine Excess & 15 & $13(86.67)^{*}$ & $2(13.33)$ & $9(60.00)^{*}$ & $6(40.00)$ \\
\hline$\chi^{2}$ & & 7.173 & & 11.227 & \\
\hline$p$ & & .028 & & .004 & \\
\hline
\end{tabular}

Note. ${ }^{*}$ In comparison to the pregnant women with iodine sufficiency, $p<.05$

\section{Discussion}

Iodine is one of indispensable trace elements for human growth and development, and it is one of main ingredients for the synthesis and release of thyroid hormones. Generally, $80 \%-90 \%$ of iodine needed by everyone comes from food, $10 \%-20 \%$ comes from drinking water and about $5 \%$ comes from the air. ${ }^{[3]}$ When the iodine intake in the human body changes, the absorption capacity of the thyroid to iodine will change adaptively. For instance, if iodine is adequate, only $10 \%$ more or less of iodine will be absorbed by the thyroid. However, if iodine is deficient, the proportion of iodine absorbed by the thyroid will reach up to $80 \%$, meanwhile, the half-life of iodine in the blood will be shortened with the thyroid activated. ${ }^{[4]}$ Thyroid function is closely related to human iodine nutritional status. Nevertheless, pregnancy is a special physiological process, and thyroid function differs significantly during different periods of pregnancy with the increase of estrogens and hCG and the influence of other related factors. The detection rate of thyroid dysfunction in pregnant women has been increasing in recent years. Studies by Wolfberg et al. ${ }^{[5]}$ show that Wolff-Chaikoff effect is the main mechanism that leads to low T4 syndrome and hypothyroidism in pregnant women, and it is an intrinsic protective effect of the thyroid tissues. When the iodine concentration in the thyroid tissues reaches up to a certain level, the iodination of thyroglobulin and the synthesis of thyroid hormones will be delayed even stopped. However, if maternal iodine intake is excessive and then the iodine concentration is ongoing high for a while, Wolff-Chaikoff effect will disappear, and then the iodination of thyroglobulin 
and the synthesis of thyroid hormones will not only return to normal, but also the synthesis of thyroid hormones will be enhanced, ${ }^{[6]}$ leading to iodine-induced hyperthyroidism. Pregnant women have an increasing demand for iodine, and they are prone to iodine deficiency. When the concentration of iodine in the blood is lower than a certain level, the synthesis of thyroid hormones is relatively insufficient, and the body changes adaptively, i.e., maternal thyroid tissues can capture more iodine, leading to compensatory hyperplasia or expansion and inducing or aggravating goiter. ${ }^{[7]}$ Some scholars have studied and found that low iodine intake may cause thyroid oxidative stress, impair the thyroid tissues and lead to hypothyroidism. ${ }^{[8]}$ However, in this research, it is found that there is no obvious correlation of the iodine nutritional status to thyroid function in pregnant women after correlation analysis. It may result from the small number of specimens and the limited study area.

The research results show that the rates of iodine deficiency in pregnant women in the early, the middle and the late trimesters of pregnancy are $36.67 \%, 66.67 \%$ and $40.00 \%$ respectively, i.e., iodine deficiency happens to pregnant women during each period of pregnancy, which may be related to the fact that all subjects live in Baotou area for quite a long time. Baotou area, far away from the sea, is located in the inland at an elevation of 1,000 meters, so that the contents of iodine both in the air and the drinking water are relatively lower. Even though the transportation industry is developing rapidly and vigorously at present, pregnant women in this area have less iodine-containing seafood in comparison to the pregnant women in the coastal area. Meanwhile, it is also because pregnant women reduce the intake of iodized salt during the cooking process in order to prevent gestational hypertension, more than that, some pregnant women choose non-iodized salt when cooking. It may also be related to the cooking method, for example, adding salt before cooking can also lead to the loss of iodide ions in the salt. The comparison in the rate of iodine deficiency among all periods of pregnancy has shown that the rate of iodine deficiency in the pregnant women in the middle trimester of pregnancy is obviously higher than that in the pregnant women both in the early and the late trimesters of pregnancy. It is because the prolonging of pregnant weeks and consequent pregnancy reactions result in lower dietary intake or dietary bias and lead to the obvious decrease of dietary intake for iodine. It is also probable that the capacity of body circulation in pregnant women in the middle trimester is increased and blood perfusion in the renal tissues is enhanced, so that the filtration of glomeruli to iodine is improved and the release of iodine from the renal tissues is increased, leading to iodine deficiency in the maternal body. In addition, the rates of iodine sufficiency in pregnant women in the early, the middle and the late trimesters of pregnancy are $40.00 \%, 26.67 \%$ and $40.00 \%$ respectively, and the rates of iodine excess are $23.33 \%, 6.66 \%$ and $20.00 \%$. There is no significant difference in the proportions of iodine sufficiency and iodine excess in pregnant women among all periods of pregnancy. Iodine excess in pregnant women may result from the excessive addition of iodized salt during the cooking process and the relatively higher intake of iodine-containing seafood. The rates of thyroid dysfunction in pregnant women with iodine deficiency, iodine sufficiency and iodine excess have been statistically analyzed, and the results show that no matter in the pregnant women with iodine deficiency or iodine excess, the rate of thyroid dysfunction is obviously higher than that in the pregnant women with iodine sufficiency, with a statistically significant difference. It is indicated that the urinary iodine concentration during pregnancy is associated with thyroid dysfunction. Therefore, it is recommended to consider the determination of the urinary iodine concentration as one of routine examination items and include it into pregnancy tests, and then give an individualized guide on the intake of iodine-containing food according to the examination results, in order to prevent pregnant women from the impairment caused by less or higher iodine intake.

The results in this research show that no matter in the pregnant women with iodine deficiency or iodine excess, the abnormality rate of antibodies is obviously higher than that in the pregnant women with iodine sufficiency, with a statistically significant difference, which conforms to the prospective research conducted by Yuanbin Li et al. ${ }^{[9]}$ In their research, the results show that there is a U-shape curve correlation of the urinary iodine concentration to the positivity of thyroid autoantibodies TPOAb and TgAb. It is also found in this research that the urinary iodine concentration in pregnant women is positively correlated to the positivity of thyroid autoantibody TPOAb, which is also consistent with the research conducted by Yuanbin Li et al. ${ }^{[9]}$ However, in our research, the urinary iodine concentration in pregnant women is not associated with thyroid autoantibody $\mathrm{TgAb}$, which differs from the research result from Yuanbin Li. The positivity of thyroid antibodies is one of main risk factors for autoimmune thyroid diseases. The research conducted by Burgi et al. ${ }^{[10]}$ shows that the increase of the urinary iodine concentration can stimulate autoimmune thyroiditis by increasing the expression of autologous TPOAb and the iodination of thyroglobulin. Multiple researches have shown that thyroid autoantibodies can enter into the fetal body by way of maternal body placenta - fetus, resulting in thyroid diseases in fetus and neonates, such as transient hypothyroidism. ${ }^{[11-15]}$ Besides, some foreign scholars also find that thyroid autoantibodies 
can directly damage the placenta tissues, meanwhile, zona pellucida shares some similar antigenic components with the thyroid, which may cause immune cross-reactions and lead to adverse pregnancy outcomes. ${ }^{[16]}$ Therefore, it is necessary for pregnant women to receive the examination of the urinary iodine concentration when taking the screening of thyroid function and antibodies, in order to reduce the occurrence of autoimmune thyroid diseases during pregnancy and cut down fetal developmental disorder and dysfunction caused by the entrance of autoantibodies into the fetal body.

In conclusion, it is supposed to advocate health education on iodine nutrition to pregnant women, and conduct the dy- namic monitoring of the urinary iodine concentration and the screening of thyroid function and autoantibodies during pregnancy, so as to adjust the intake of iodine-containing food according to the urinary iodine concentration and scientifically give an individualized guide on the iodine intake. The purpose is not only to prevent iodine deficiency caused by less iodine intake, but also avoid iodine excess resulting from unchecked iodine supplementation, so as to prevent from the occurrence of thyroid diseases during pregnancy and guarantee maternal and child health.

\section{CONFLiCTS OF INTEREST DisClosure}

The authors declare they have no conflicts of interest.

\section{REFERENCES}

[1] WHO/UNICEF/ICCIDD. Assessment of iodine deficiency disorders and monitoring their elimination: A guide for programme managers (Third Edition). Geneva: WHO; 2007. 32-34p.

[2] Chinese Society of Endocrinology, Chinese Medical Association; Chinese Society of Perinatology, Chinese Medical Association. Guideline on diagnosis and management of thyroid diseases during pregnancy and postpartum. Chinese Journal of Endocrinology and Metabolism. 2012; 28(2): 354-371.

[3] Swanson CA, Pearce EN. Iodine insufficiency: a global health problem? Adv Nutr. 2013; 4(5): 533-535. PMid:24038248. https : //doi.org/10.3945/an.113.004192

[4] Loh JA, Wartofsky L, Jonklaas J, et al. The magnitude of increased levothyroxine requirements in hypothyroid pregnant women depends upon the etiology of the hypothyroidism. Thyroid. 2009; 19(3): 269275. PMid:19265498. https://doi.org/10.1089/thy . 2008.0 413

[5] Wolfberg AJ, Nagey DA. Thyroid disease during pregnancy and subsequent congenital anomalies. Article presented at the annual meeting of the Society for Maternal-Fetal Medicine in New Orleans. 2002; 47: 986 .

[6] Sang ZN, Zhang SF, Wei W, et al. Effects of excess iodine intake on maternal thyroid function and pregnancy outcomes. Acta Nutrimenta Sinica. 2011; 33(5): 472-475.

[7] Wu L, Yu JC, Kang WM, et al. Iodine nutrition and thyroid diseases. Acta Academiae Medicinae Sinicae. 2013; 35(4): 363-368.

[8] J Burton GJ, Jauniaux E. Place oxdatibe: from miscarriage to Preeclampsia. Sov Gynecol Inbest. 2004; 11(6): 342-352. PMid:15350246. https://doi.org/10.1016/j.jsgi.2004.03 .003
[9] Li YB, Teng WP, Shan ZY, et al. Prospective study on the effect of pregnancy on thyroid autoantibodies.//Chinese Society of Endocrinology, Chinese Medical Association. Proceedings of 2008 Endocrine and Metabolic Diseases Seminar Series \& Young and Middleaged English Forum - Proceedings of Endocrine and Metabolic Diseases Seminar Series, Harbin, 2008. Beijing: Chinese Society of Endocrinology, Chinese Medical Association.

[10] Burgi H. Iodine excess. Best Pract Res Clin Endocrinol Metab. 2010; 24(1): 107-115. PMid:20172475. https://doi.org/10.1016/j. beem. 2009.08.010

[11] Lian XL, Bai Y, Xu YH, et al. Effects of Maternal Hyperthyroidism and Antithyroid Drug Therapy on Thyroid Function of Newborn Infants. Acta Academiae Medicinae Sinicae. 2005; 27(6): 756-760.

[12] Xu C, Yan WS, Hu J. Outcomes survey of thyroid function screening for pregnant women in the first trimester and their neonates. Chinese Journal of Medicine. 2018; 53(3): 310-313.

[13] Zhou Y, Zhang HY, Wang L. Effect of late-pregnancy subclinical hypothyroidism on maternal immunologic function and pregnancy outcomes. Chinese Clinical Doctor. 2019; 47(2): 228-230.

[14] Wang JH, Li XH, Li L. Effect of subclinical hypothyroidism on pregnancy outcomes and its thyroid hormone alternative medicine. Chinese Journal of the Frontiers of Medical Science (Electronic Version). 2019; 11(4): 81-84.

[15] Dong X, Wang K, Wang M. Intelligence level and the influence factors in offsprings of subclinical hypothyroidism during pregnancy. China Medicine. 2018; 13(9): 1394-1397.

[16] Mecacci F, Parretti E, Cioni R, et al. Thyroid autoimmunity and its association with non-organ-specific antibodies and subclinical alterations of thyroid function in women with a history of pregnancy loss or preeclampsia. J Reprod Immunol. 2000; 46(1): 39-50. https ://doi .org/10.1016/S0165-0378(99)00055-8 